\title{
Development of Fault Management Dispatcher Training Simulator for TDAS in Korea
}

\author{
In-Yong Seo, Sang-Ok Kim, Bok-Nam Ha \\ Smart Energy Lab, KEPCO Research Institute, Munjiro, Yuseong, Daejeon, Korea \\ Email: iyseo@kepri.re.kr
}

Received 2013

\begin{abstract}
A fault management dispatcher training simulator for large-scale Distribution Automation System (TDAS) is developed to train operators in distribution control center. This simulator is composed of independent simulation server and operator consoles and can be used for network analysis, network operation, fault management and evaluation. TDAS DB is duplicated online to the simulation server keeping the data security. The system can model distribution network penetrated with distributed generations (DG) using the real data from the TDAS DB. Network fault scenarios are automatically generated by calculating fault current and generating fault indicators. Also, manual entry of cry wolf alarm is available. Moreover, operation solution for scenario of fault isolation and service restoration is generated automatically so that trainee can check their operation result. Operator actions during training session are saved and can be played back as well as displayed on one-line diagram pictures.
\end{abstract}

Keywords: Distribution Automation System; Fault Management Dispatcher Training Simulator; Distributed Generation

\section{Introduction}

With high penetration of distribution generation (DG), power system operations are becoming increasingly complicated and critical. There is a need to provide more directed power system operation training than that provided by on-the-job experience. Dispatcher training simulator (DTS) can realistically simulate electricity system in the actual production, so that the trainees are familiar with the system, to develop its capability of handling various incidents.

The handling of network failures (e.g. short circuits, earth faults) offers a lot of possibilities for automation. The main tasks are fault location isolation and service restoration (FISR). The network operator needs excellent knowledge about the controlled network area to perform these tasks efficiently. Without efficient aids by automation, the operator has to achieve and to keep these skills by spending a lot of time with a dispatcher training simulator.

Lots of research have been concentrated on DTS related to Energy Management System (EMS) and Supervisory Control and Data Acquisition (SCADA) [1-3] but not many papers for fault management DTS (FM-DTS) were reported [4,5]. In this paper a fault management system for DG interconnected distribution networks is described which automates the fault localization and the determination of isolation and restoration measures to relief the operator from these demanding tasks during network failures.

KEPCO has been operating 186 TDAS for urban areas. As can be seen in Figure 1, it has a dual server, three human-machine interface (HMI) terminals, and a frontend processor (FEP). It adopted DNP 3.0 protocol over fiber optic cable, dedicated metallic line, wireless data packet, trunked radio system, etc.

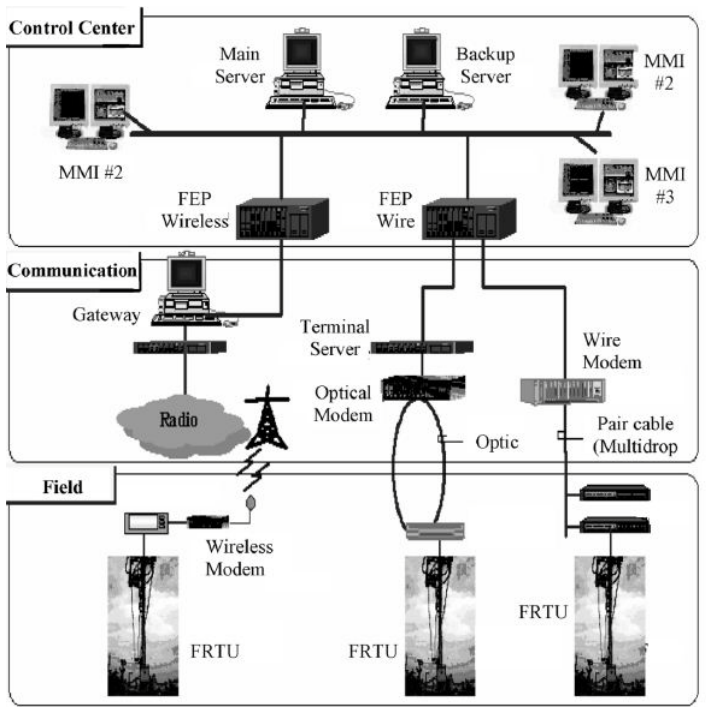

Figure 1. Central control system of TDAS. 


\section{Fault Management DTS}

\subsection{Configurations and Features}

The developed FM-DTS shown in Figure 2 has three main subsystems:

Simulation Server: This server saves coded TDAS DB, scenario, solution of FISR, trainee's train history, trainee's switch operation actions, user information and score statistics.

Simulation Console: This console uses simulation software and displays for generating fault scenarios with which users can perform self-training. Several application programs are running in the console which perform short circuit analysis and power flow analysis, and generating fault event and solution of FISR. Also, it converts fault scenarios to the format for competition console.

Competition Console: This console is used for practical test and written test. All operator's actions are logged so that instructors can review their mistakes during the practical test. Written test program is an internet based test (IBT) that includes short-answer question and multiple choice about operation of distribution networks. Test results for both tests are automatically scored and analyzed for statistics by the system, and transferred to the simulation server.

This simulator, from software implementation point of view comprises of the following modules
a) DB Manager
b) Simulator Manager
c) Scenario Manager
d) Application Programs
e) Operation History Manager
f) HMI (NZed, agOLD, AgWorks, Host-server)

\subsection{Online Duplication of DB for Simulation}

TDAS is one of most important national security facilities in Korea. TDAS DB should be secure in any case but real DB is needed for training the service restoration of distribution networks. The DB is duplicated in two ways, i.e. real time and periodic request by the DB handler.

Figure 3 shows configuration of DB online duplication, and the procedures for duplication is described in the following:

Step 1. Make a backup DB and checksum file in OA link server

Step 2. Encode the backup DB

Step 3. Copy and save the encoded DB and checksum file to simulation server

Step 4. Copy and decode the encoded DB and checksum file to simulation console

Step 5. Generate a checksum file for the decoded DB in simulation console.

Step 6. Compare the checksum files in step 1 and 5. If those files are identical, we can assure integrity of TDAS.

Step 7. Delete all DB data in simulation console at the end of simulation program automatically.

\subsection{Simulator Manager}

Simulator manager is installed in the simulation console and has following functions;

- Execution of host-server and HMI

- Creation of distribution line fault scenarios

- Creation of substation fault scenarios

- Management of scenarios

- Inquiry of train history

- Management of user access

- Close of simulation program

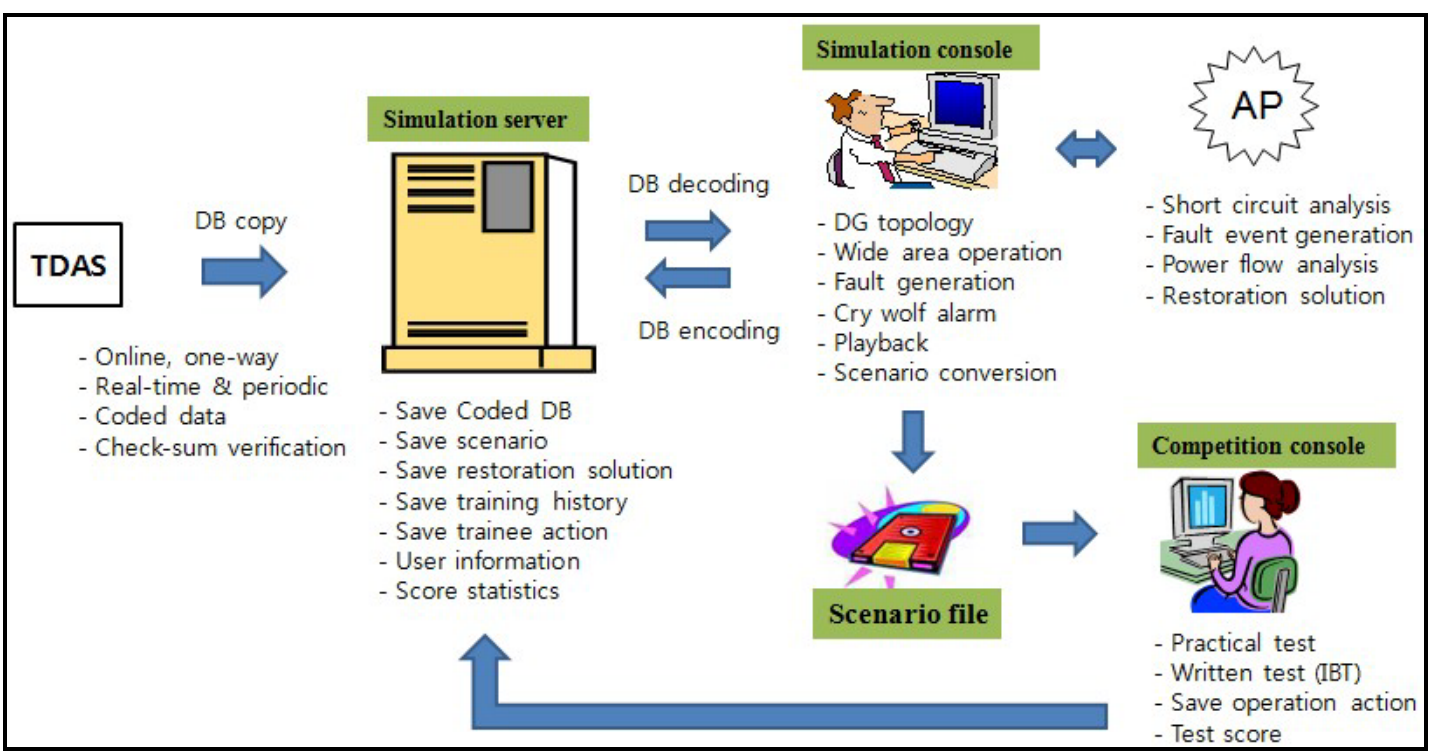

Figure 2. Configuration of fault management DTS. 


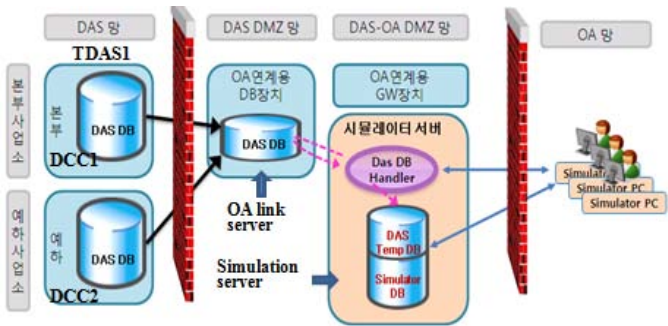

Figure 3. Configuration of DB online copy.

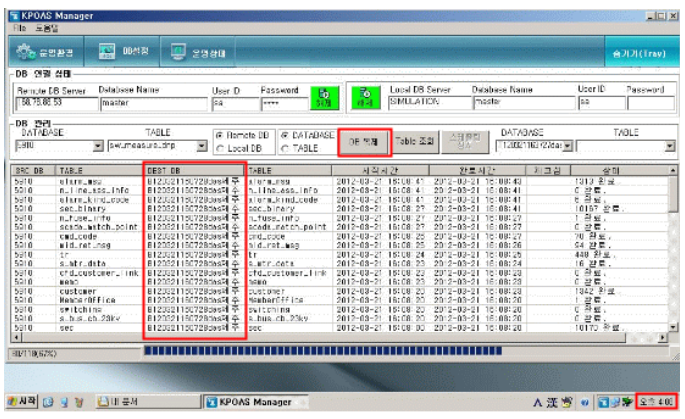

Figure 4. DB handler program.

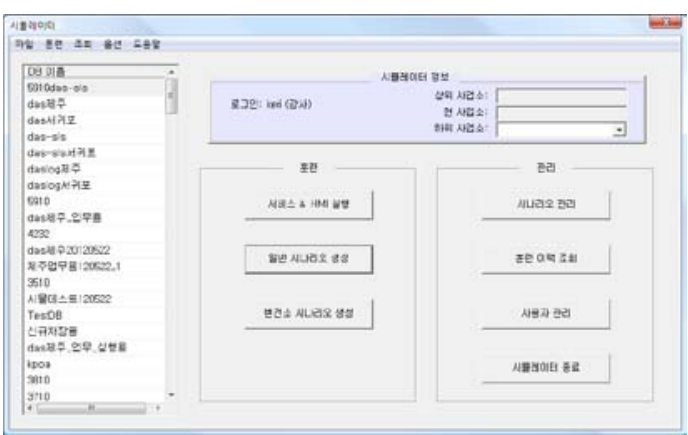

Figure 5. Simulator manager user interface.

\subsection{Scenario Manager}

Scenario manager produces fault scenarios and cry wolf alarm shown in Figure 6 and $\mathbf{7}$ respectively. The fault types for scenarios are 1 phase ground, 2 phase ground, 3 phase short. Fault location can be designated using the switch and distance from the switch. Also, this can generates scenarios for different protection coordination cases such as complete, incomplete and impossible protection coordination.

Figure 8 represents a scenario generated by the scenario manager. Using this scenario, trainees can start fault isolation and service restoration train.

\subsection{Wide Area Operation}

In TDAS, wide area operation (WAO) is available which means operators in distribution control center (DCC) can manage switches in distribution line which belong to regional control center (RCC) by transferring control authority. Each control center including DCC and RCC has its own middleware (M/W) and naming service.

To realize WAO in the FM-DTS, M/W configuration is changed as shown in Figure 9 in which the M/W manages several naming services.

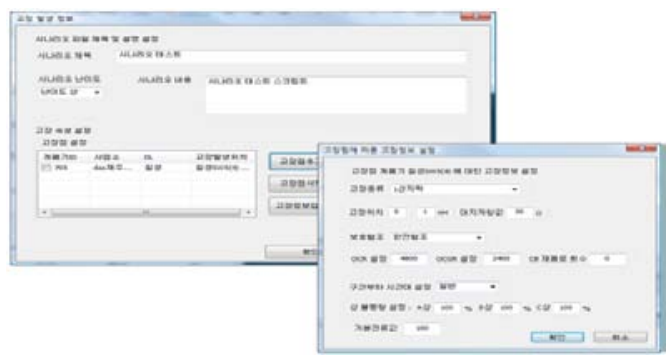

Figure 6. Scenario generation.

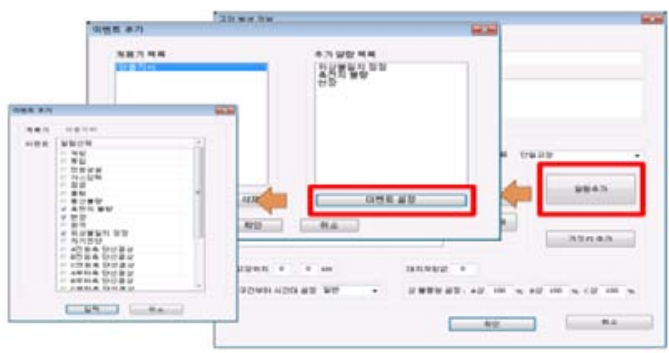

Figure 7. Cry wolf alarm addition.

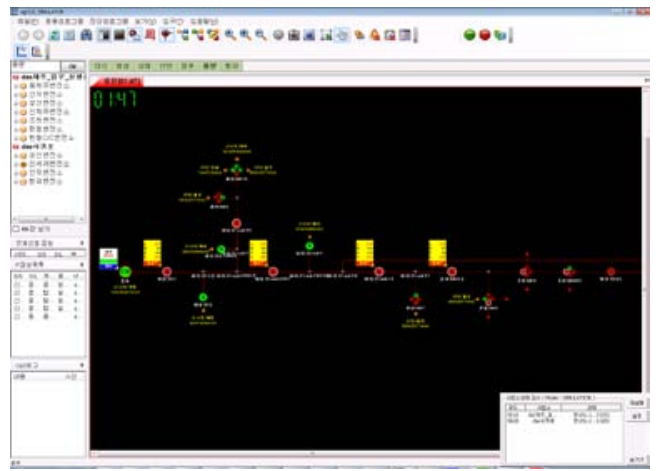

Figure 8. One Scenario case.

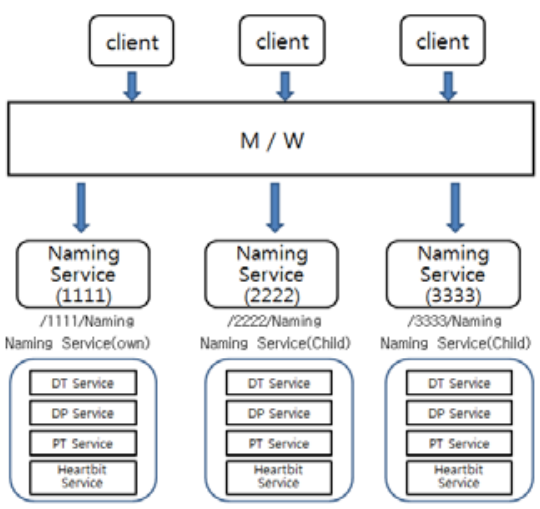

Figure 9. Configuration of FM-DTS M/W. 


\section{Application Programs of FM-DTS}

\subsection{Short Circuit Analysis Program}

This application calculates short circuit current in DG interconnected distribution network system based on IEC 60909 standards. Several kinds of fault currents at different time instants after fault occurrence can be generated. This module is composed of Network Connectivity Processor (NCP) and Short Circuit Analysis (SCA) [6]. The flowchart for fault current calculation is shown in Figure 10.

NCP: This application performs the function changing the state of system topology, presented to node state on the kind of breaker or switch, into the form of 'bus' using the open or close state of those kinds. Also, the topological island and the equipment state(Live or Dead) are classified.

SCA: Based on positive, negative and zero sequence impedance, the fault current for the each section of the power distribution system is calculated according to IEC 60909 in case of fault occurrence.

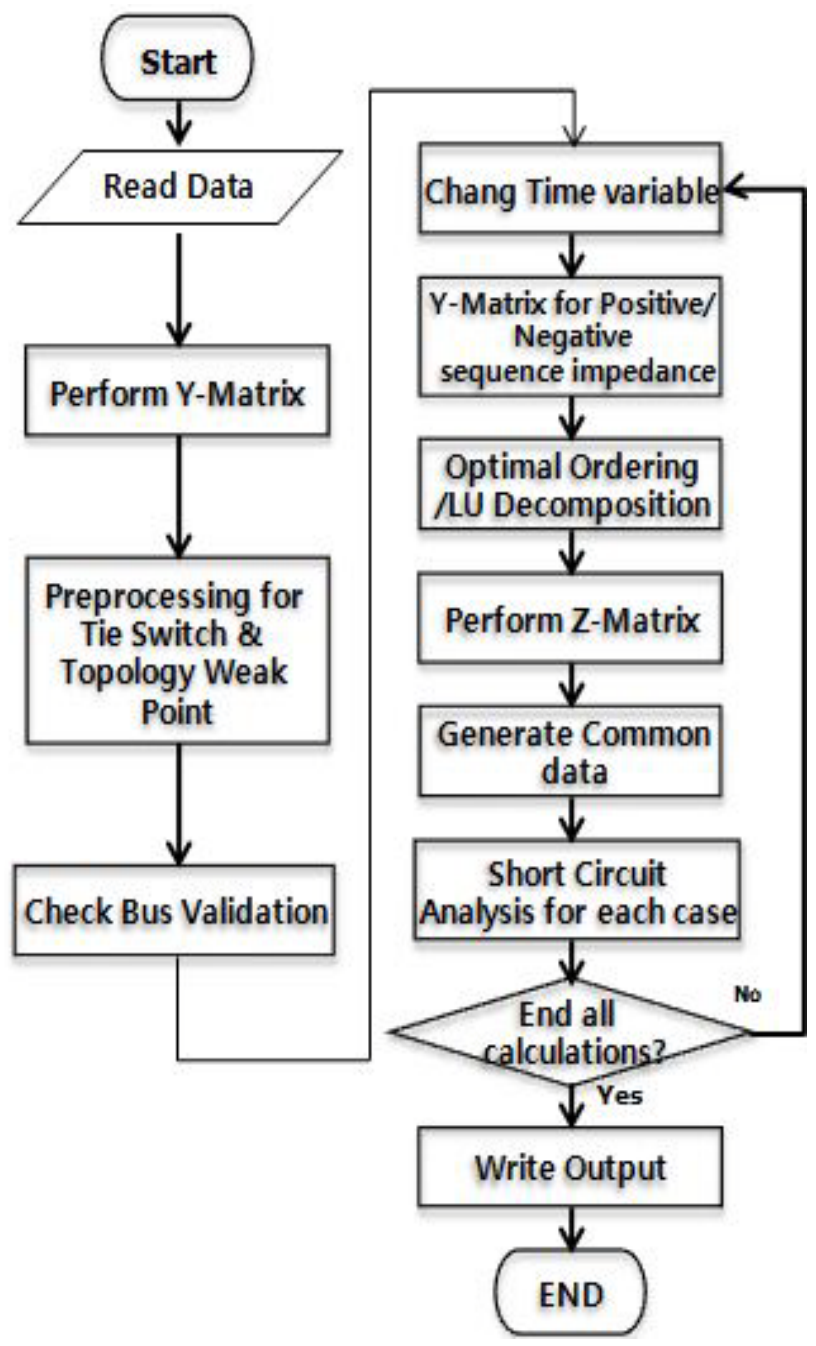

Figure 10. Flowchart of short-circuit current calculation.

\subsection{Automatic Service Restoration Program}

This program which utilized the algorithm in [7] generates operation procedures for fault isolation and system restoration so that trainee can perform self-training referring to those procedures. The proposed restoration strategy consists of two steps: Candidate Set Generation and Fuzzy Decision Making. The fault is classified in 6 classes as shown in Figure 11, i.e., Self Restoration, Single Group Restoration (SGR), Double Group Restoration (DGR), Triple Group Restoration (TGR), Single Group \& Level-2 Load Transfer (SGRLT), Double Group \& Level-2 Load Transfer (DGRLT). The Fuzzy rule makes decisions to minimize the number of switching, live load transfer, and to balance the load of the network [8].

Figure 12 shows FISR solution produced by this program (a) and operation actions manipulated by trainer (b). The solution is separated into several fault areas to be restored because the operation list is long. And it shows 3 solution candidates from the highest priority for each area.

\subsection{Topology Considering DG}

Network topology for FM-DTS is developed to prevent islanding operation of DGs in distribution network. If any switch installed in source side changes to open, all the vacuum circuit breakers (VCB) of DG after the switch are tripped, while in case of DAS only energized status of line after the switch will be dead.
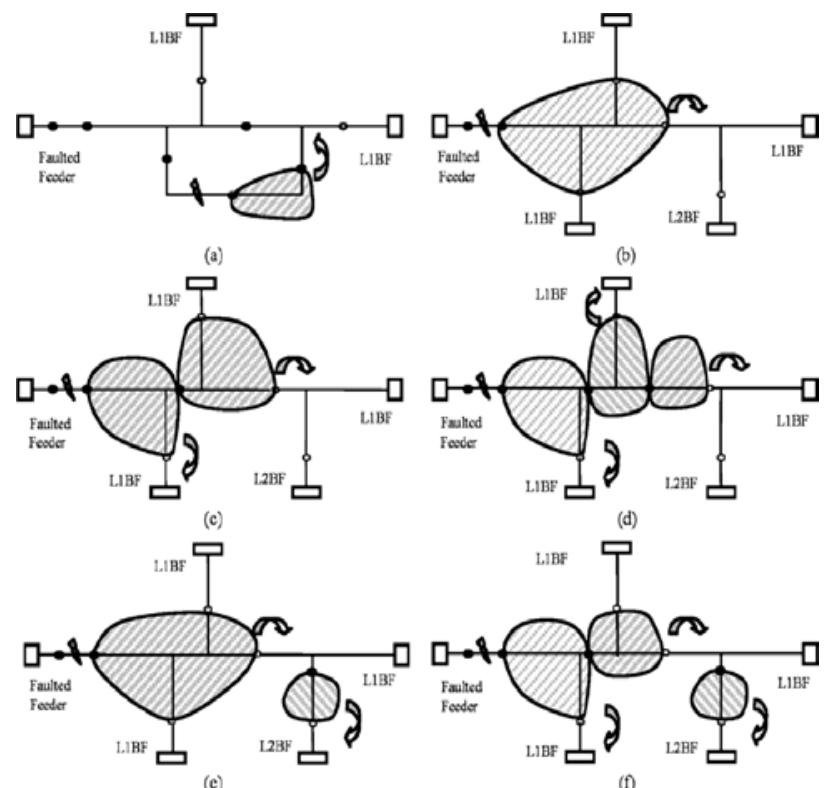

$$
\begin{array}{|cc|}
\hline \begin{array}{c}
\text { O Open Switch } \\
\text { L1BF : Level-1 Backup Feeder }
\end{array} & \text { L2BF : Level-2 Backup Feeder } \\
\hline
\end{array}
$$

Figure 11. Six basic restoration schemes (a)Self-restoration. (b)SGR (c)DGR (d)TGR. (e)SGR_LT (f)DGR_LT. 

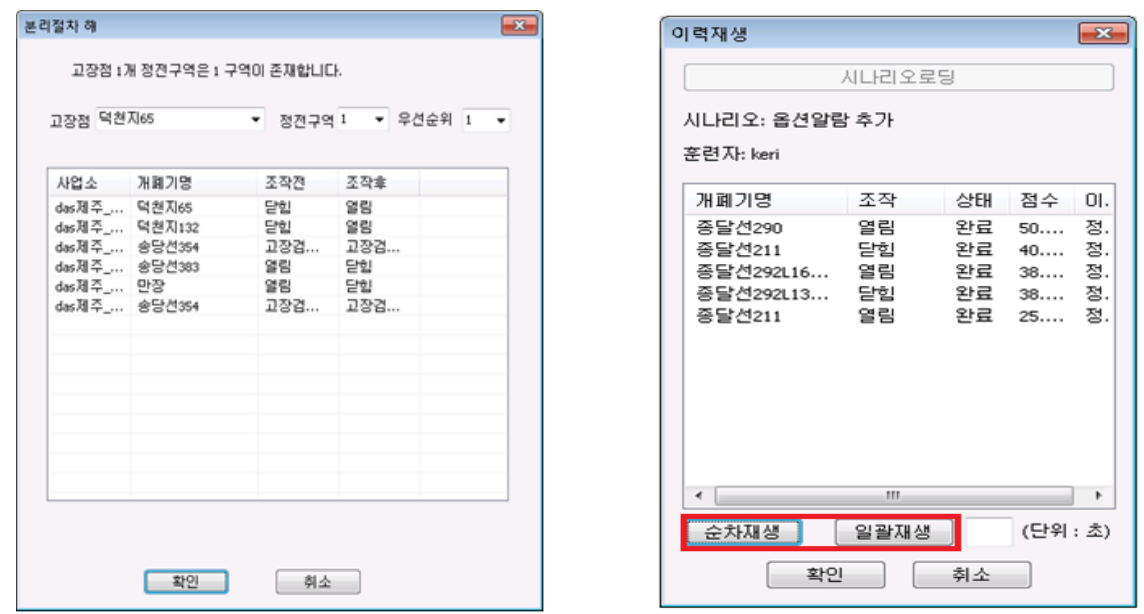

Figure 12. FLIS procedure (a) Solution (b) Playback.

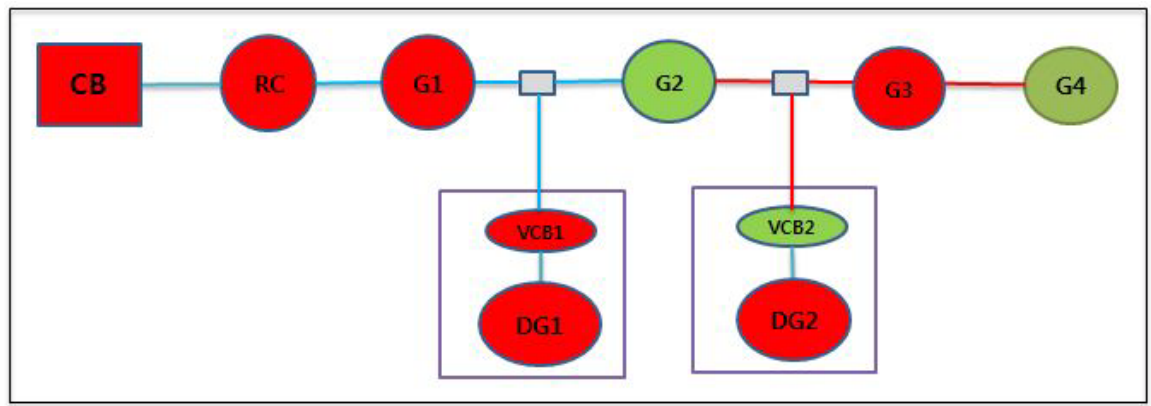

Figure 13. An example of network topology.

Figure 13 shows an example of DG topology. When switch G2 is open, only VCB2 changes to trip and lines after G2 will be de-energized, while the status of DG2 is unchanged.

\section{Operation}

The simulator is implemented on a HP workstation with Windows 2003 Server operating system and MS-SQL Server 2008. The MMI is XP window based one with MS-SQL Server 2008.

Figure 14 shows a simulation flow chart of this simulator. A brief description of running of the simulator is as follows:

To start the simulation process, the simulator reads the static data of the network over which simulation is to be performed.

Users input all fault information that is need to define the fault such as type of fault, fault location, resistance to ground, etc. With these information, application program calculates fault currents, and generates fault indicator (FI) at each switches automatically.

After scenario is generated, instructor can modify the scenario by adding cry wolf alarms like communication error, battery low of FRTU, switch open etc.

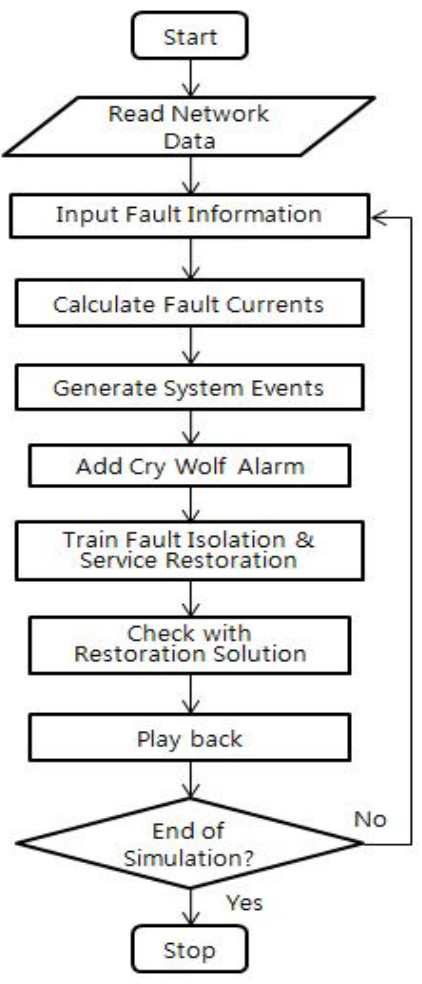

Figure 14. Flowchart of simulation. 
Now, trainer can start fault isolation and service restoration by manipulating the switches on one-line diagram.

Trainer can refer to solution of FISR during and after the train session which is created by application program.

After self-training, trainer can play back the operation actions what he did during the train session in step by step or batch mode.

Once session is finished, trainer can save the scenario and start another session by generating new scenarios. tion.

The above process gets repeated till the end of simula-

\section{Conclusion}

A fault management dispatcher training simulator for large-scale Distribution Automation System in Korea is developed to train operators who work in distribution control center. This simulator can be used for training of network analysis, network operation, fault management and evaluation. The simulator can model distribution network penetrated with DG using the real data from the TDAS DB. Network fault scenarios are automatically generated by calculating fault current and generating fault events. Also, manual entry of cry wolf alarm is available. Moreover, operation solution for FISR scenario is generated automatically so that trainee can check their operation result. Operator actions during training session are saved and can be played back as well as displayed on one-line diagram pictures.

This simulator will be installed nation-wide next year. It will be very helpful to train operators resulting in shortening the outage time as well as number of human errors.

\section{REFERENCES}

[1] P. I. Hwang, S. J. Ahn, S. I. Hur, Y. T Yoon, S. I. Moon, "Adaptive Step Size Method for the Power System Model of Dispatcher Training Simulator," Proceedings of the 2010 Power and Energy Society General Meeting, July 2010, pp. 1-7.

[2] S. V. Vadari, M. J. Montstream, H. B. Ross, “An Online Dispatcher Training Simulator Function for Real-Time Analysis and Training," IEEE Transactions on Power Systems, Vol. 10, No. 4, 1995, pp. 1798-1804

[3] D. Bolborici, "ExpDTS - Hybrid Dispatcher Training Simulator Expert System for operator assistance and evaluation," Proceedings of the 2009 Power Tech, July 2009, pp. 1-5.

[4] R. Apel, C Jaborowicz and R. Kussel, "Fault Management in Electrical Distribution Networks," Proceedings of CIRED2001, June 2001, Conference Publication No. 482.

[5] Krishna. K. Agarwal, J. S. Candlish, T. L. Carney, “Automatic Fault Location and Isolation System for the Electric Traction Overhead Lines," Proceedings of the 2002 ASME/IEEE Joint Rail Conference, April 2002, pp. 117-122.

[6] C. H. Chu, Y. S. Yun, S. C. Kwon and I. K. Song, "Design of Application Model for Network Analysis in Smart Distribution Management," Proceedings of the 21st International Conference and Exhibition on Electricity Distribution, Frankfurt, 6-9 June 2011, Paper 0224.

[7] S. H. Lee, S. I. Lim and B. S. Ahn, "Service restoration of primary distribution systems based on fuzzy evaluation of multi-criteria," IEEE Transactions on Power Systems, Vol. 13, No. 3, 1998, pp. 1156-1163.

[8] S. I. Lim, S. J. Lee, M. S. Choi, D. J. Lim and B. N. Ha, "Service Restoration Methodology for Multiple Fault Case in Distribution Systems," IEEE Transactions on Power Systems, Vol. 21, No. 4, 2006, pp. 1638-1644. 